\title{
Adaptation requirements due to anatomical changes in free-breathing and deep- inspiration breath-hold for standard and dose-escalated radiotherapy of lung cancer patients
}

Sibolt, Patrik; Ottosson, Wiviann; Sjöström, David; Larsen, Christina; Behrens, Claus F.

Published in:

Acta Oncologica

Link to article, DOI:

10.3109/0284186X.2015.1062543

Publication date:

2015

Document Version

Publisher's PDF, also known as Version of record

Link back to DTU Orbit

Citation (APA):

Sibolt, P., Ottosson, W., Sjöström, D., Larsen, C., \& Behrens, C. F. (2015). Adaptation requirements due to anatomical changes in free-breathing and deep-inspiration breath-hold for standard and dose-escalated radiotherapy of lung cancer patients. Acta Oncologica, 54(9), 1453-1460.

https://doi.org/10.3109/0284186X.2015.1062543

\section{General rights}

Copyright and moral rights for the publications made accessible in the public portal are retained by the authors and/or other copyright owners and it is a condition of accessing publications that users recognise and abide by the legal requirements associated with these rights.

- Users may download and print one copy of any publication from the public portal for the purpose of private study or research.

- You may not further distribute the material or use it for any profit-making activity or commercial gain

- You may freely distribute the URL identifying the publication in the public portal 
This article was downloaded by: [Patrik Sibolt]

On: 16 August 2015, At: 02:46

Publisher: Taylor \& Francis

Informa Ltd Registered in England and Wales Registered Number: 1072954 Registered office: 5 Howick Place, London, SW1P 1WG

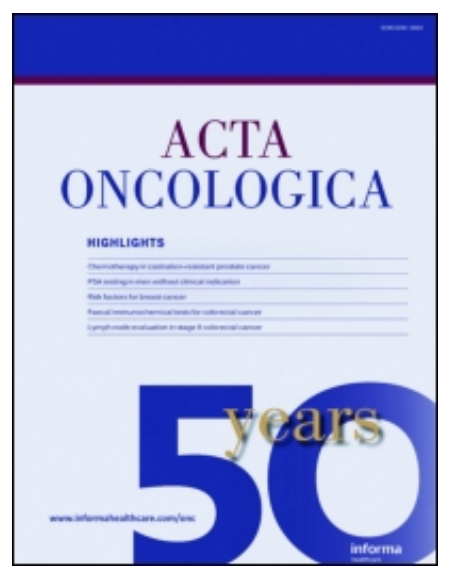

CrossMark

\section{Acta Oncologica}

Publication details, including instructions for authors and subscription information:

http:// www. tandfonline.com/loi/ionc20

\section{Adaptation requirements due to anatomical changes in free-breathing and deep-inspiration breath-hold for standard and dose-escalated radiotherapy of lung cancer patients}

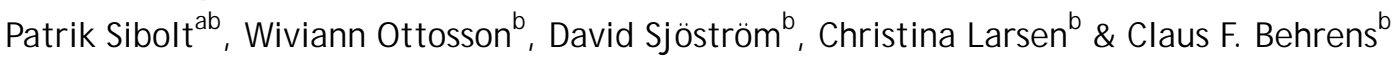 \\ ${ }^{a}$ Center for Nuclear Technologies, Technical University of Denmark, DTU Risø Campus, \\ Roskilde, Denmark \\ ${ }^{\mathrm{b}}$ Department of Oncology, Radiotherapy Research Unit, Herlev Hospital, University of \\ Copenhagen, Herlev, Denmark \\ Published online: 14 Aug 2015.
}

To cite this article: Patrik Sibolt, Wiviann Ottosson, David Sjöström, Christina Larsen \& Claus F. Behrens (2015): Adaptation requirements due to anatomical changes in free-breathing and deep-inspiration breath-hold for standard and dose-escalated radiotherapy of lung cancer patients, Acta Oncologica

To link to this article: http:// dx. doi.org/ 10.3109/ 0284186X.2015.1062543

\section{PLEASE SCROLL DOWN FOR ARTICLE}

Taylor \& Francis makes every effort to ensure the accuracy of all the information (the "Content") contained in the publications on our platform. However, Taylor \& Francis, our agents, and our licensors make no representations or warranties whatsoever as to the accuracy, completeness, or suitability for any purpose of the Content. Any opinions and views expressed in this publication are the opinions and views of the authors, and are not the views of or endorsed by Taylor \& Francis. The accuracy of the Content should not be relied upon and should be independently verified with primary sources of information. Taylor and Francis shall not be liable for any losses, actions, claims, proceedings, demands, costs, expenses, damages, and other liabilities whatsoever or howsoever caused arising directly or indirectly in connection with, in relation to or arising out of the use of the Content.

This article may be used for research, teaching, and private study purposes. Any substantial or systematic reproduction, redistribution, reselling, loan, sub-licensing, systematic supply, or distribution in any form to anyone is expressly forbidden. Terms $\&$ Conditions of access and use can be found at http:// www.tandfonline.com/page/terms-and-conditions 


\title{
Adaptation requirements due to anatomical changes in free-breathing and deep-inspiration breath-hold for standard and dose-escalated radiotherapy of lung cancer patients
}

\author{
PATRIK SIBOLT ${ }^{1,2}$, WIVIANN OTTOSSON ${ }^{2}$, DAVID SJÖSTRÖM $^{2}$, \\ CHRISTINA LARSEN ${ }^{2} \&$ CLAUS F. BEHRENS ${ }^{2}$ \\ ${ }^{1}$ Center for Nuclear Technologies, Technical University of Denmark, DTU Risø Campus, Roskilde, Denmark and \\ ${ }^{2}$ Department of Oncology, Radiotherapy Research Unit, Herlev Hospital, University of Copenhagen, Herlev, \\ Denmark
}

\section{ABSTRACT}

Background. Radiotherapy of lung cancer patients is subject to uncertainties related to heterogeneities, anatomical changes and breathing motion. Use of deep-inspiration breath-hold (DIBH) can reduce the treated volume, potentially enabling dose-escalated (DE) treatments. This study was designed to investigate the need for adaptation due to anatomical changes, for both standard (ST) and DE plans in free-breathing (FB) and DIBH.

Material and methods. The effect of tumor shrinkage (TS), pleural effusion (PE) and atelectasis was investigated for patients and for a CIRS thorax phantom. Sixteen patients were computed tomography (CT) imaged both in FB and DIBH. Anatomical changes were simulated by CT information editing and re-calculations, of both ST and DE plans, in the treatment planning system. PE was systematically simulated by adding fluid in the dorsal region of the lung and TS by reduction of the tumor volume.

Results. Phantom simulations resulted in maximum deviations in mean dose to the GTV-T $\left.(<\mathrm{D}\rangle_{\mathrm{GTV}-\mathrm{T}}\right)$ of $-1 \%$ for $3 \mathrm{~cm} \mathrm{PE}$ and centrally located tumor, and $+3 \%$ for TS from $5 \mathrm{~cm}$ to $1 \mathrm{~cm}$ diameter for an anterior tumor location. For the majority of the patients, simulated PE resulted in a decreasing $\langle\mathrm{D}\rangle_{\text {GTV-T }}$ with increasing amount of fluid and increasing $\langle\mathrm{D}\rangle_{\mathrm{GTV}-\mathrm{T}}$ for decreasing tumor volume. Maximum change in $\langle\mathrm{D}\rangle_{\mathrm{GTV}-\mathrm{T}}$ of $-3 \%(3 \mathrm{~cm}$ PE in FB for both ST and DE plans) and $+10 \%$ ( $2 \mathrm{~cm}$ TS in FB for DE plan) was observed. Large atelectasis reduction increased the $<\mathrm{D}>_{\text {GTV-T }}$ with $2 \%$ for FB and had no effect for DIBH.

Conclusion. Phantom simulations provided potential adaptation action levels for PE and TS. For the more complex patient geometry, individual assessment of the dosimetric impact is recommended for both ST and DE plans in DIBH as well as in FB. However, DIBH was found to be superior over FB for DE plans, regarding robustness of $\langle\mathrm{D}\rangle_{\mathrm{GTV}-\mathrm{T}}$ to TS.

Lung cancer is one of the most common cancer diseases worldwide [1-3]. Unfortunately lung cancer patients also suffer from a high mortality rate and the local control for patients undergoing radiotherapy as a part of their treatment is poor. Escalating the radiation dose to the tumor could lead to an increased cure rate [4]. Even if this statement is debatable in the light of the results of the RTOG0617 study $[5,6]$, the idea of dose-escalated (DE) radiotherapy of lung cancer patients is generally supported and emerging dose escalation studies, having higher focus on quality assurance and keeping the dose to normal tissues as low as for ST treatments, should proceed [7].

In radiotherapy of lung cancer patients, extensive margins are added to the tumor volume in order to account for uncertainties related to baseline shifts, delineation, organ motion and respiration $[8,9]$. This results in large irradiated volumes of adjacent normal tissue, limiting escalation of the dose to the tumor. Setup margins in the thorax region can be reduced

Correspondence: P. Sibolt, Center for Nuclear Technologies, Technical University of Denmark, DTU Risø Campus, Fredriksborgvej 399, Building 201, 4000 Roskilde, Denmark. E-mail: pasi@dtu.dk 
by moving from bony match on the spine towards direct match on the soft tissue of the lung tumor [10]. Further margin reduction is conceivable by synchronizing the radiation delivery with tumor motion [11]. Use of deep-inspiration breath-hold (DIBH) reduces the tumor motion and can potentially enable DE treatments of lung cancer patients, without increasing the severe radiation-induced side effects of organs at risk (OARs) [12,13].

Radiotherapy of lung cancer patients is, however, also subject to uncertainties related to heterogeneities and anatomical changes. As margins are minimized and dose to the tumor is conformed and escalated, adaptation of the treatment plan due to anatomical changes could become more crucial. Previous studies demonstrate that changes in dose due to anatomical changes have the largest effect on the delivered dose to the tumor [14]. One reason being the lung density alteration that arises with the appearance of anatomical changes, such as atelectasis, pneumonitis, pleural effusion (PE) and tumor shrinkage (TS). While previous studies have investigated the effect of anatomical changes and need for adaptive strategies in ST fractionated treatment of lung cancer patients in free-breathing (FB) [14,15], there is a lack of studies investigating the potential need for adaptation in $\mathrm{DE}$ treatment of lung cancer patients in DIBH.

This study is designed to investigate the need for adaptation due to lung density alteration as a result of PE, TS or atelectasis, for both ST and DE plans in $\mathrm{FB}$ and DIBH.

\section{Material and methods}

\section{Data and treatment preparations}

In order to perform simulations in a reproducible, well defined geometry, a three-dimensional computed tomography (3DCT) of the CIRS thorax phantom (CIRS Inc., Norfolk, VA, USA) was acquired. Spherical gross tumor volumes (GTV-Ts) with a diameter of $5 \mathrm{~cm}$ were delineated in the anterior (A), central (C) and posterior (P) part of the left lung insert, and given Hounsfield units (HUs) corresponding to water. OARs, clinical target volumes (CTV-Ts) and planning target volumes (PTV-Ts) were defined and ST plans were produced, for all situations.

Furthermore, 16 locally advanced non-small cell lung cancer (NSCLC) patients scheduled for curative radiotherapy, between December 2012 and July 2014, were enrolled. One 4DCT in FB and one 3DCT in DIBH, both with intravenous contrast, were acquired prior to, in the middle of and after the course of treatment. Target volumes and OARs, both in $\mathrm{FB}$ and DIBH, were delineated separately on each CT dataset by an oncologist in a treatment planning system (TPS) (Eclipse, Varian Medical Systems, Palo Alto, CA, USA) [10]. Dose calculations of ST (utilized for treatment on Varian 2300 iX linear accelerators [16]) and DE volumetric modulated arc therapy (VMAT) plans were carried out in the same TPS, using the AAA calculation algorithm [17].

Additional information regarding the clinical protocol, patient characteristics, scanning procedures, delineations and treatment planning is provided in the Supplementary Appendix (available online at: http://informahealthcare.com/doi/abs/10.3109/0284 186X.2015.1062543).

\section{Pleural effusion}

The dosimetric effect of PE was simulated for ST plans in FB and three different tumor locations in the CIRS thorax phantom (Figure 1a). Simulations of PE in patients were carried out on the pre-treatment CT scans by adding 1,2 or $3 \mathrm{~cm}$ fluid in the dorsal region of the lung (patient in supine position) (Figure 1b), resulting in three new image sets per patient and scanning technique. Simulations were carried out by altering the HUs in the PE region to correspond to water. PE was realistically simulated in the sense that no adjustment was made to the fact that the posterior wall of the lung was not in the same vertical position for the entire longitudinal extension of the patient. This implies that the amount of fluid varied over the longitudinal length of the lung, including the tumor region and depending on the

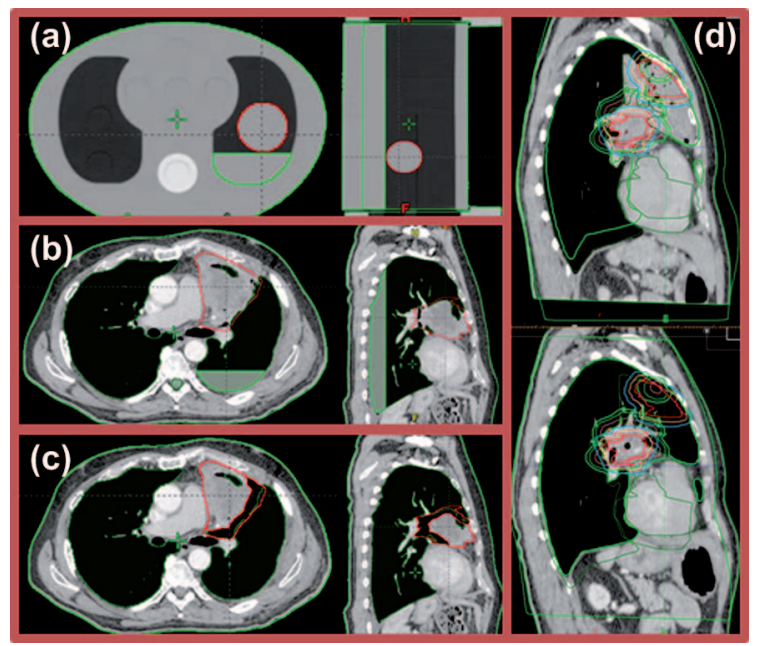

Figure 1. Examples of simulated anatomical changes: a) $3 \mathrm{~cm}$ pleural effusion for the centrally located tumor in the CIRS thorax phantom, b) $3 \mathrm{~cm}$ pleural effusion in a patient, c) tumor shrinkage in the form of $1 \mathrm{~cm}$ radius reduction in a patient, and d) large atelectasis disappearance between pre-treatment (upper) and post-treatment (lower) CT. Colors available online. 
patients' lung geometry as well as tumor location. Original ST and DE plans were re-calculated on the new image sets, preserving the original monitor units (MU).

\section{Tumor shrinkage}

TS was simulated for ST plans and the three tumor locations in the CIRS thorax phantom. Simulations of TS in patients were carried out on the pre-treatment CT scans by reductions of the GTV-T radius of the tumor regions adjacent to the lung, both in FB and DIBH (Figure 1c). Thus, parts of tumor extending into the mediastinum, thoracic wall or adjacent to the spinal cord were not reduced. TS was simulated stepwise from a $0.5 \mathrm{~cm}$ to a $2.0 \mathrm{~cm}$ reduction, with a step size of $0.5 \mathrm{~cm}$, by altering HUs in the subtracted volume to correspond to the surrounding lung tissue, resulting in four new image sets per patient and scanning technique. New CTV-Ts and PTV-Ts were re-generated in each new image set. One patient was excluded due to atelectasis at the time of pre-treatment CT. Furthermore, not all patients had GTV-Ts large enough to enable shrinkage in all steps. Original ST and DE plans were recalculated on the new image sets, preserving the MU from the original plans.

\section{Atelectasis}

One patient had atelectasis enclosing the GTV-T at the planning stage (Figure 1d upper). The atelectasis regressed and finally disappeared during the course of treatment (Figure 1d lower). Pre-treatment FB
4DCT and DIBH 3DCT were rigidly registered to corresponding CTs acquired post-treatment, according to a soft tissue tumor position verification procedure currently in practice at our clinic. OARs and target volumes were re-delineated on the posttreatment CTs. The dosimetric effect of atelectasis was investigated by re-calculation of the original ST and DE plans in FB and DIBH on the posttreatment $\mathrm{CT}$ scans, preserving the $\mathrm{MU}$ from the original plans.

\section{Statistical analysis}

Statistical analysis was carried out in MATLAB, including the Statistics Toolbox 8.3 (R2013b), (The MathWorks, Natick, MA, USA). The aim was to discover potentially statistically significant differences between the impacts of anatomical changes in DIBH and FB as well as between ST and DE plans. Bonferroni corrected two-sided Wilcoxon signed rank tests were applied to test the medians of the data, for each level of anatomical change. Results were considered statistically significant at $p \leq 0.004$ and $p \leq 0.003$, for $\mathrm{PE}$ and TS, respectively.

\section{Results}

\section{Pleural effusion}

Phantom simulations resulted in a reduction of the mean dose to the GTV-T $\left.(<\mathrm{D}\rangle_{\text {GTV-T }}\right)$ with increasing amount of $\mathrm{PE}$ for all tumor positions (Figure 2), with a maximum decrease of $1.3 \%$ for the centrally located tumor. For anteriorly and
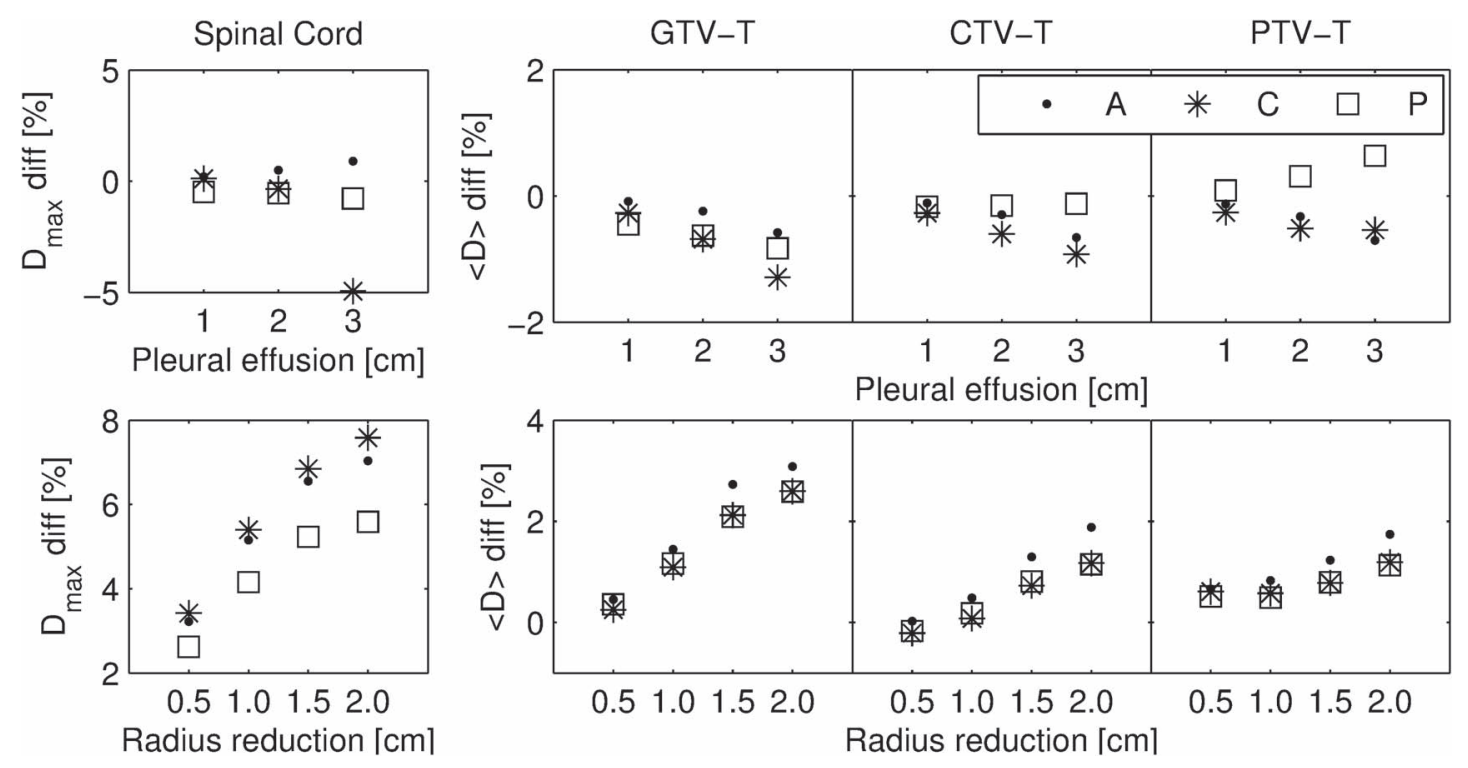

Figure 2. Impact of pleural effusion (upper row) and tumor shrinkage (lower row) on Dmax to the spinal cord as well as $<\mathrm{D}>{ }_{\mathrm{GTV}} \mathrm{T}$ $<\mathrm{D}\rangle_{\mathrm{CTV}-\mathrm{T}}$ and $\left\langle\mathrm{D}>_{\mathrm{PTV}-\mathrm{T}}\right.$ for simulations carried out in a CIRS dynamic thorax phantom (locations: A, anterior; C, central; P, posterior). 
centrally located tumors, this trend was also visible for the mean dose to the CTV-T $\left.(<\mathrm{D}\rangle_{\mathrm{CTV}-\mathrm{T}}\right)$ and the mean dose to the PTV-T $\left.(<\mathrm{D}\rangle_{\text {PTV-T }}\right)$. For the posteriorly positioned tumor only a slight decrease of the $\langle\mathrm{D}\rangle_{\text {CTV-T }}$ was observed, while the $\langle\mathrm{D}\rangle_{\text {PTV-T }}$ increased.

For the majority of the patients, simulated PE resulted in decreasing $\langle\mathrm{D}\rangle_{\text {GTV-T }}$ with increasing $\mathrm{PE}$ (Figure 3). Up to $2.8 \%$ decrease was observed for $3 \mathrm{~cm} \mathrm{PE}$ in FB for both ST and DE plans (Patient 6). For the largest amount of PE, median decrease in $<\mathrm{D}\rangle_{\text {GTV-T }}$ among patients was similar in DIBH $(-0.3 \%$ for both ST $[-2.4 \% ; 0.7 \%]$ and DE $[-2.3 \%$; $0.6 \%$ plans $)$ and in $\mathrm{FB}(-0.3 \%[-2.8 \% ; 0.5 \%]$ for ST and $-0.4 \%[-2.8 ; 0.6]$ for DE plans). Some patients (9, 12 and 15) presented no change in $\langle\mathrm{D}\rangle_{\text {GTV-T }}$ with changing amount of $\mathrm{PE}$, and for a few patients (7, 10 and 13) $<\mathrm{D}>_{\text {GTV-T }}$ even increased with increasing amount of PE (Figure 3).

The Bonferroni corrected two-sided Wilcoxon signed rank test resulted in no statistically significant differences, when considering the effect of PE on $<\mathrm{D}\rangle_{\text {GTV-T }}$, between FB and DIBH or between ST and DE plans.

Additional results for the effect of $\mathrm{PE}$ on the maximum dose $\left(\mathrm{D}_{\text {max }}\right)$ to the spinal cord is provided in the Supplementary Appendix available online at: http://informahealthcare.com/doi/abs/10.3109/ 0284186X.2015.1062543.

\section{Tumor shrinkage}

Phantom simulations of TS resulted in increasing $\langle\mathrm{D}\rangle_{\text {GTV-T }},\langle\mathrm{D}\rangle_{\text {CTV-T }}$ and $\langle\mathrm{D}\rangle_{\text {PTV-T }}$ with decreasing GTV-T, for all tumor locations (Figure 2). TS had less effect on the $\langle\mathrm{D}\rangle_{\text {CTV-T }}$ than on the $\langle\mathrm{D}\rangle_{\text {GTV-T }}$, and even smaller impact on the $\langle\mathrm{D}\rangle_{\text {PTV-T }}$ A maximum increase of the $\langle\mathrm{D}\rangle_{\text {GTV-T }}$ by $3.1 \%$ was observed for the anteriorly located tumor. A difference $\geq 1 \%$ in mean dose was reached at a radius reduction of $1 \mathrm{~cm}$ for the GTV-T, and $1.5 \mathrm{~cm}$ reduction for the CTV-T and PTV-T.

Similar to phantom simulations, increasing $<\mathrm{D}>_{\text {GTV-T }}$ for decreasing tumor volume was observed for the majority of the patients, regardless of motion management technique and fraction dose (Figure 4), with a maximum increase of $10.4 \%$ (DE plan in FB for Patient 3). For the largest amount of TS, median increase in $\langle\mathrm{D}\rangle_{\text {GTV-T }}$ among patients was smaller in DIBH $(2.6 \%$ [0.8\%; $4.1 \%]$ for ST and 2.5\% [-3.6\%; $6.9 \%$ ] for DE plans) than in $\mathrm{FB}(2.6 \%$ [0.6\%; $4.7 \%]$ for ST and 5.1\% ([.6\%; 10.4\%] for DE plans). In a few cases $\langle\mathrm{D}\rangle_{\text {GTV-T }}$ decreased with decreasing tumor volume (DE plan in DIBH for Patients 6, 9 and 11, and ST plan in DIBH for Patients 5 and 9).

For TS, Bonferroni corrected Wilcoxon signed rank testing on the percentage difference in $<\mathrm{D}_{\text {GTV-T }}$, resulted in statistically significant differences, at $0.5 \mathrm{~cm}$ radius reduction of the GTV-T, between ST and DE plans for both DIBH $(p<0.001$; median increase of $0.4 \%[-0.4 \% ; 1.1 \%]$ for ST and $1.1 \%[-0.2 \% ; 6.4 \%]$ for $\mathrm{DE}$ plans) and $\mathrm{FB}$ $(\mathrm{p}<0.001$; median increase of $0.5 \%$ [0.0\%; $0.7 \%$ ] for ST and $1.7 \%[0.0 \% ; 3.8 \%]$ for DE plans). However, for $1 \mathrm{~cm}$ radius reduction, statistical significance was only observed for corresponding differences in $\mathrm{FB}(\mathrm{p}=0.002$; median increase of $1.1 \%$ $[0.4 \% ; 1.8 \%]$ for ST and $3.4 \%[0.6 \% ; 5.5 \%]$ for DE plans). For all other cases, no statistically significant differences were observed.

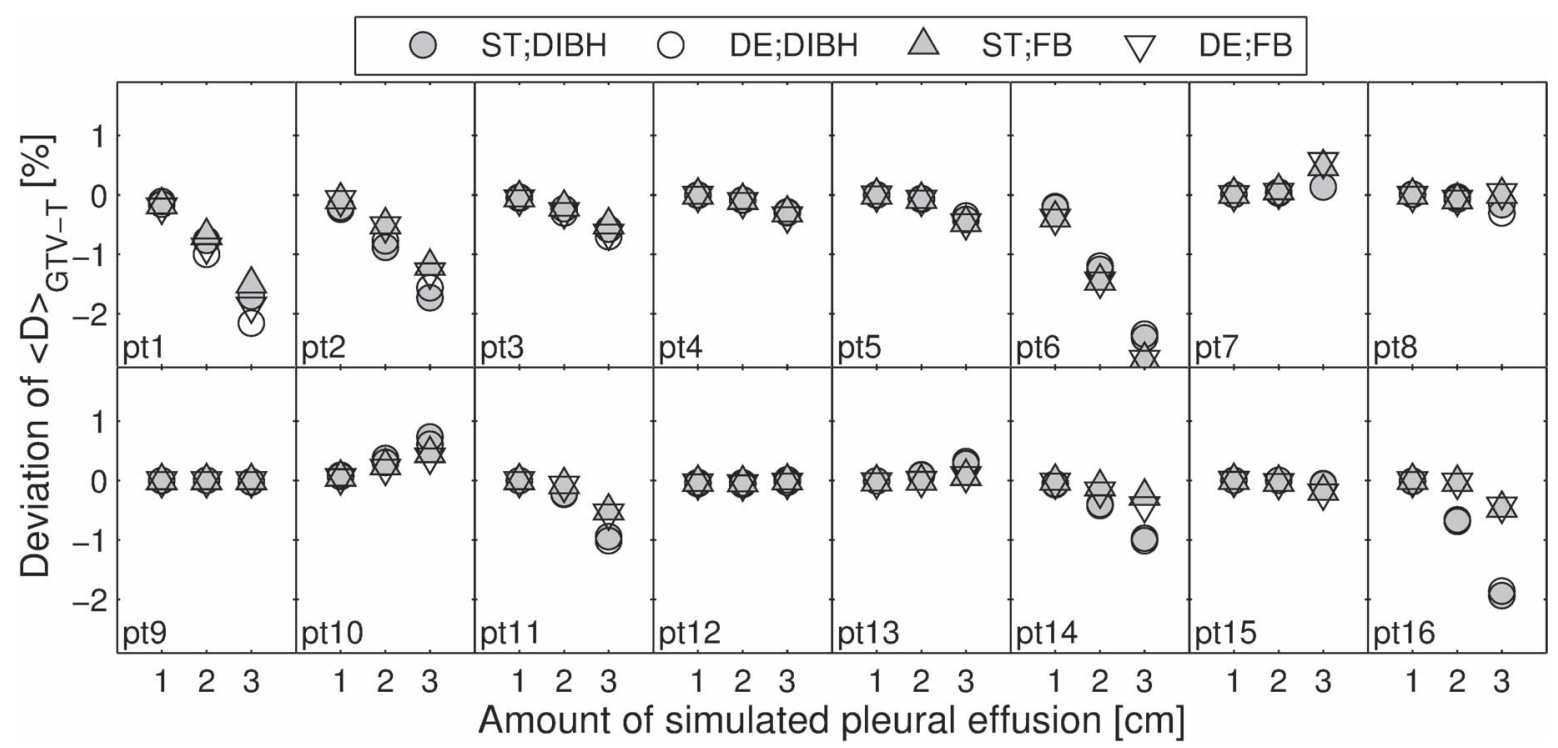

Figure 3. Impact of pleural effusion on $\langle\mathrm{D}\rangle_{\text {GTV-T }}$ for all 16 patients and combinations of scanning technique and fractionation. 


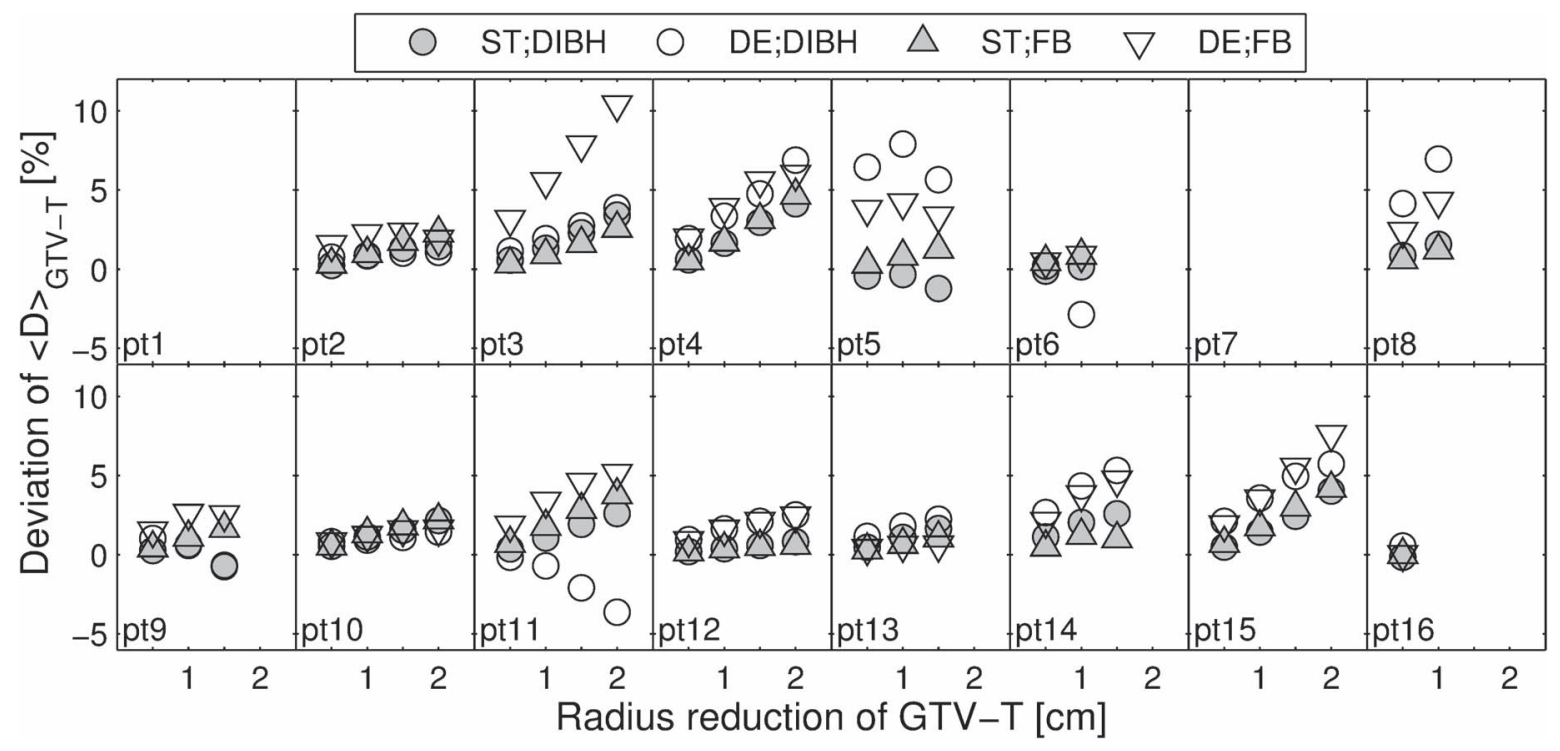

Figure 4. Impact of tumor shrinkage on $\langle\mathrm{D}\rangle_{\mathrm{GTV}-\mathrm{T}}$ for all 16 patients and combinations of scanning technique and fractionation.

Spinal cord results for TS are provided in the Supplementary Appendix available online at: http:// informahealthcare.com/doi/abs/10.3109/0284186X. 2015.1062543.

\section{Atelectasis}

In addition to atelectasis reduction during the course of treatment for Patient 1 (Figure 1d), the total GTV-T decreased by $43.8 \%$ and $24.8 \%$ for FB and DIBH, respectively. These changes increased the mean dose of the total GTV-T for the ST plan with $2.4 \%$ in $\mathrm{FB}$ and $0.2 \%$ in DIBH. Corresponding numbers for the DE plans were an increase of $1.9 \%$ and a decrease of $5.2 \%$ for $\mathrm{FB}$ and $\mathrm{DIBH}$, respectively. A larger decrease in minimum dose to the target was observed in DIBH (45.3\% for ST and 53.8\% for DE plan) than in FB (0.1\% for ST and 3.5\% for DE plan). Spinal cord results are available in the Supplementary Appendix available online at: http:// informahealthcare.com/doi/abs/10.3109/0284186X. 2015.1062543 .

\section{Discussion}

The dosimetric impacts of PE, TS and atelectasis on VMAT treatment of NSCLC patients were investigated, both for ST and DE treatments in FB as well as in DIBH. For the clinically realistic ranges of PE and TS considered in the current study, both phantom (Figure 2) and patient simulations (Figures 3 and 4, Supplementary Figures 1 and 2, available online at: http://informahealthcare.com/ doi/abs/10.3109/0284186X.2015.1062543) indicate that TS have larger dosimetric impact than PE, when considering both targets and the spinal cord.

For the majority of the patients a decreasing $<\mathrm{D}>_{\text {GTV-T }}$ was observed for increasing amount of $\mathrm{PE}$, while spinal cord $\mathrm{D}_{\max }$ increased. This general decrease in target dose is in concordance with previous studies $[14,15]$. The exceptions from this are in most cases due to the location of the tumor. A posteriorly positioned tumor will in some cases get surrounded by fluid during PE, resulting in a build-up effect that is greater than the attenuating effect. This is, e.g. indicated by the increase in $\langle\mathrm{D}\rangle_{\text {CTV-T }}$ and $<\mathrm{D}>_{\text {PTV-T }}$ of the posteriorly located tumor in the phantom simulations. The few patient cases where almost no change in $\langle\mathrm{D}\rangle_{\text {GTV-T }}$ was present during PE simulations could be explained by the tumor being located in the superior region of the lung where no PE was present (located outside the photon beam). The variation in tumor shapes, sizes and locations of the patient cohort, do also to some extent explain the spread in the resulting deviations from the original $\left\langle\mathrm{D}>_{\text {GTV-T }}\right.$ No apparent difference was observed, when considering robustness to $\mathrm{PE}$, in using DIBH or FB, neither for ST or DE plans, also supported by the lack of statistically significant differences, when considering $\langle\mathrm{D}\rangle_{\text {GTV-T }}$ Any conclusion regarding potential advantage, in terms of robustness to $\mathrm{PE}$, in utilizing $\mathrm{DIBH}$ or $\mathrm{FB}$ when moving towards DE plans, would require larger data sets to strengthen the statistics.

TS mostly resulted in an increase in $\langle\mathrm{D}\rangle_{\text {GTV-T }}$ for decreasing tumor volume, complying with the finding of Schmidt et al. [14], who for ST plans observed increases of $\langle\mathrm{D}\rangle_{\text {CTV-T }}$ of up to $4.0 \%$ when TS occurred. Similarly, also spinal cord $\mathrm{D}_{\max }$ 
increased with decreasing tumor volume for the majority of the patients. These effects were furthermore observed when simulating TS in a phantom, regardless of tumor location. However, phantom simulations were carried out by isotropic reduction of the tumor radius, while TS in patients was carried out by only altering the GTV-T adjacent to lung tissue. Furthermore, the patient population consisted of a variation of original GTV-T volumes and locations, affecting the tumor reductions and contributing to the observed inter-patient variation, regarding the differences in $\langle\mathrm{D}\rangle_{\text {GTV-T }}$ Original tumor volume can be of importance, as, e.g. reduction of already small tumors surrounded by lung tissue will increase the effect of lack of charged particle equilibrium. However, a larger patient cohort is necessary in order to make a statistically significant statement regarding this dependency. Alternatively, actual anatomical changes appearing during the course of treatment could be utilized, as in previous studies [14,15]. Nevertheless, statistically significant differences in $<\mathrm{D}>_{\text {GTV-T }}$ were observed between ST and DE plans for $0.5 \mathrm{~cm}$ and $1 \mathrm{~cm}$ simulated TS when treating in FB. For DIBH, statistical significance between ST and DE plans was only observed for a $0.5 \mathrm{~cm}$ reduction. This indicates that: 1) DE plans are potentially inferior to ST plans in terms of robustness to TS, when considering $\langle\mathrm{D}\rangle_{\text {GTV-T }}$; and 2) $\mathrm{DIBH}$ potentially can reduce the impact of TS for DE treatments. This could be explained by the smaller original GTV-T volume when utilizing DIBH, compared to FB [13].

The low variation in the effect on the $\langle\mathrm{D}\rangle_{\text {GTV-T }}$ for $\mathrm{TS}<1 \mathrm{~cm}$ radius reduction could explain the statistically significant differences observed when comparing ST and DE plans. The same variation generally increases with increasing anatomical changes and was observed to be larger in comparisons between motion management techniques than fractionation schemes. However, for both PE andTS, statistical analysis of the difference between DIBH and FB resulted in decreasing p-values with increasing anatomical changes, even if never reaching statistical significance, and smaller p-values were generally observed for DE than for ST plans. The median deviations in $\langle\mathrm{D}\rangle_{\text {GTV-T }}$ for the largest anatomical changes indicate that $\mathrm{DIBH}$ has a potential superiority when considering TS (Figure 4), while similar responses to PE were observed (Figure 3).

When considering atelectasis, varying results regarding changes in doses to the GTV were observed amongst the different scanning techniques and fractionations in the one patient where atelectasis disappeared during treatment. However, GTV-T also decreased during the course of treatment (larger decrease observed in FB than in DIBH). Together with registration and delineation uncertainties, this could explain the dosimetric variations observed.

In general, the present study indicates a potential benefit in utilizing $\mathrm{DIBH}$ instead of $\mathrm{FB}$ during $\mathrm{DE}$ radiotherapy of NSCLC patients, regarding robustness to anatomical changes. However, it does not take into account any differences in inter- or intrafractional uncertainties during setup and delivery of VMAT treatment in DIBH or FB. Furthermore, as positron emission tomography (PET) is necessary for DE treatment, adaption of treatment plans may require a new PET [18]. In addition to the PET signal being sensitive to tissue response after radiotherapy, performing a reproducible PET at the stage of adaption may be a challenge, and is not trivial, as the PET-active volume may be deformed, systematically moved, or new PET-active volumes may be present [19]. The patients enrolled in the current study were only PET imaged in FB. Thus, in order to minimize the bias between $\mathrm{DE}$ plans in $\mathrm{FB}$ and $\mathrm{DIBH}$, the dose escalation was applied to the entire GTV-T volume in both image sets. The impact of this on the results in the current study is considered small.

Standard action levels are difficult to define. If $1 \%$ change in $\langle\mathrm{D}\rangle_{\text {GTV-T }}$ would be considered as the action level required, phantom data indicate that action should be taken for $\mathrm{PE}$ of $\geq 3 \mathrm{~cm}$ thickness and TS of $\geq 1 \mathrm{~cm}$ radius reduction. This was not confirmed by the patient data, where a larger variation in changes with PE and TS was observed. However, PE results are in concordance with Møller et al. [15], who observed PE for 13 of 163 studied patients and deviations in $\left\langle\mathrm{D}>_{\text {PTV-T }} \geq 1 \%\right.$ in four cases with $\geq 2$ cm PE.

Randomly occurring anatomical changes of the sort presented in this study can most likely not be accounted for by extending margins. Thus, an adaptive strategy is necessary where the changes during the course of treatment are monitored for each patient individually. Møller et al. [15] have presented results of a random pattern in the appearance and disappearance of anatomical changes supporting this statement. Rapid adaption to anatomical changes (e.g. daily adaptive plan selection) has previously been proven beneficial for normal tissue sparing in sites, such as bladder [20]. A similar approach, together with the reduction in treatment volume from utilizing $\mathrm{DIBH}$, is appealing for sparing normal tissue in lung cancer radiotherapy. Similarly to the current study, an approach of density alterations, based on daily cone beam computed tomography (CBCT) image information, could be utilized in order to alter the original CT, on which re-calculation of the original 
treatment plan could be carried out. Preferably, deformable image registration would be utilized in order to transfer anatomical information from the CBCT to the original CT image. However, there are known issues required to be solved before having a reliable deformable image registration tool effective for evaluating lung cancer radiotherapy over the course of treatment [21]. It should be mentioned that there are limitations in the AAA calculation algorithm when calculating dose in heterogeneous situations [22-24], and Monte Carlo simulations could potentially render different results [25].

In conclusion, phantom simulations have provided information about potential action levels for when adaptation to appearance of either PE or TS is required. However, for the more complex patient geometry, also tumor volume and location needs to be taken into consideration. Individual assessment of the dosimetric impact should rather be carried out instead of applying standard action levels. This is the recommendation for both $\mathrm{ST}$ and $\mathrm{DE}$ treatment plans in DIBH as well as in FB, even if the current study suggests a potential superiority in $\mathrm{DIBH}$, regarding the robustness in $\langle\mathrm{D}\rangle_{\mathrm{GTV}-\mathrm{T}}$ to anatomical changes in $\mathrm{DE}$ treatment.

\section{Acknowledgments}

The authors would like to express gratitude to the radiation oncologists, Jon A Lykkegaard Andersen and Svetlana Borissova, for the delineations carried out on each patient in this study. Furthermore Anders Mellemgaard, together with Jon A Lykkegaard Andersen and Svetlana Borissova, are acknowledged for enrolling the patients for this study. Special thanks are also given to the RTT team for executing the additional CT scans according to the imaging protocol.

Declaration of interest: The authors report no conflicts of interest. The authors alone are responsible for the content and writing of the paper.

\section{References}

[1] Ferlay J, Soerjomataram I, Ervik M, Dikshit R, Eser S, Mathers C, et al. GLOBOCAN 2012 v1. 0, Cancer incidence and mortality worldwide: IARC CancerBase No. 11. Lyon, France; IARC: 2013. [cited 2015 Mar 18]. Available from: http://globocan.iarc.fr.

[2] Engholm G, Ferlay J, Christensen N, Kejs AMT, Johannesen TB, Khan S, et al. NORDCAN: Cancer incidence, mortality, prevalence and survival in the Nordic Countries, Version 7.0 (17.12.2014). Association of the Nordic Cancer Registries. Danish Cancer Society. [cited 2015 Mar 18]. Available from: http://www.ancr.nu.
[3] Engholm G, Ferlay J, Christensen N, Bray F, Gjerstorff ML, Klint $\AA$, et al. NORDCAN-a Nordic tool for cancer information, planning, quality control and research. Acta Oncol 2010;49:725-36.

[4] Rengan R, Rosenzweig KE, Venkatraman E, Koutcher LA, Fox JL, Nayak R, et al. Improved local control with higher doses of radiation in large-volume stage III non-small-cell lung cancer. Int J Radiat Oncol Biol Phys 2004;60:741-7.

[5] Bradley JD, Paulus R, Komaki R, Masters G, Blumenschein G, Schild S, et al. Standard-dose versus high-dose conformal radiotherapy with concurrent and consolidation carboplatin plus paclitaxel with or without cetuximab for patients with stage IIIA or IIIB non-small-cell lung cancer (RTOG 0617): A randomised, two-by-two factorial phase 3 study. Lancet Oncol 2015;16:187-99.

[6] Faivre-Finn C. Dose escalation in lung cancer: Have we gone full circle? Lancet Oncol 2015;16:125-7.

[7] Belderbos J, Walraven I, van Diessen J, Verheij M, de Ruysscher D. Radiotherapy dose and fractionation for stage III NSCLC. Lancet Oncol 2015;16:e156-7.

[8] van Herk M, Remeijer P, Rasch C, Lebesque JV. The probability of correct target dosage: Dose-population histograms for deriving treatment margins in radiotherapy. Int J Radiat Oncol Biol Phys 2000;47:1121-35.

[9] Juhler-Nøttrup T, Korreman SS, Pedersen AN, Persson GF, Aarup LR, Nyström $\mathrm{H}$, et al. Interfractional changes in tumour volume and position during entire radiotherapy courses for lung cancer with respiratory gating and image guidance. Acta Oncol 2008;47:1406-13.

[10] Ottosson W, Baker M, Hedman M, Behrens CF, Sjöström D. Evaluation of setup accuracy for NSCLC patients; studying the impact of different types of cone-beam CT matches based on whole thorax, columna vertebralis, and GTV. Acta Oncol 2010;49:1184-91.

[11] Chin E, Loewen S, Nichol A, Otto K. 4D VMAT, gated VMAT, and 3D VMAT for stereotactic body radiation therapy in lung. Phys Med Biol 2013;58:749-70.

[12] Rosenzweig KE, Hanley J, Mah D, Mageras G, Hunt M, Toner S, et al. The deep inspiration breath-hold technique in the treatment of inoperable non-small-cell lung cancer. Int J Radiat Oncol Biol Phys 2000;48:81-7.

[13] Hanley J, Debois MM, Mah D, Mageras GS, Raben A, Rosenzweig K, et al. Deep inspiration breath-hold technique for lung tumors: The potential value of target immobilization and reduced lung density in dose escalation. Int J Radiat Oncol Biol Phys 1999;45:603-11.

[14] Schmidt ML, Hoffmann L, Kandi M, Møller DS, Poulsen PR. Dosimetric impact of respiratory motion, interfraction baseline shifts, and anatomical changes in radiotherapy of non-small cell lung cancer. Acta Oncol 2013;52:1490-6.

[15] Møller DS, Khalil AA, Knap MM, Hoffmann L. Adaptive radiotherapy of lung cancer patients with pleural effusion or atelectasis. Radiother Oncol 2014;110:517-22.

[16] Sjostrom D, Bjelkengren U, Ottosson W, Behrens CF. A beam-matching concept for medical linear accelerators. Acta Oncol 2009;48:192-200.

[17] Fogliata A, Nicolini G, Vanetti E, Clivio A, Cozzi L. Dosimetric validation of the anisotropic analytical algorithm for photon dose calculation: Fundamental characterization in water. Phys Med Biol 2006;51:1421-38.

[18] Møller DS, Khalil AA, Knap MM, Muren LP, Hoffmann L. A planning study of radiotherapy dose escalation of PETactive tumour volumes in non-small cell lung cancer patients. Acta Oncol 2011;50:883-8.

[19] Aerts HJ, Bosmans G, van Baardwijk AA, Dekker AL, Oellers MC, Lambin P, et al. Stability of 18 F-deoxyglucose uptake locations within tumor during radiotherapy for 
NSCLC: A prospective study. Int J Radiat Oncol Biol Phys 2008;71:1402-7.

[20] Vestergaard A, Muren LP, Lindberg H, Jakobsen KL, Petersen JB, Elstrøm UV, et al. Normal tissue sparing in a phase II trial on daily adaptive plan selection in radiotherapy for urinary bladder cancer. Acta Oncol 2014;53:997-1004.

[21] Ottosson W, Andersen JL, Borissova S, Mellemgaard A, Behrens CF. Deformable image registration for geometrical evaluation of DIBH radiotherapy treatment of lung cancer patients. J Phys Conf Ser 2014;489:012077.

[22] Van Esch A, Tillikainen L, Pyykkonen J, Tenhunen M, Helminen $\mathrm{H}$, Siljamäki S, et al. Testing of the analytical

\section{Supplementary material available online}

Supplementary Appendix, Figures 1-2 and Table I available online at: http://informahealthcare.com/ doi/abs/10.3109/0284186X.2015.1062543 anisotropic algorithm for photon dose calculation. Med Phys 2006;33:4130-48.

[23] Tillikainen L, Helminen $H$, Torsti $T$, Siljamäki S, Alakuijala J, Pyyry J, et al. A 3D pencil-beam-based superposition algorithm for photon dose calculation in heterogeneous media. Phys Med Biol 2008;53:3821-39.

[24] Robinson DM. Inhomogeneity correction and the analytic anisotropic algorithm. J Appl Clin Med Phys 2008;9:112-22.

[25] Ottosson R, Karlsson A, Behrens C. Pareto front analysis of 6 and 15 MV dynamic IMRT for lung cancer using pencil beam, AAA and Monte Carlo. Phys Med Biol 2010;55: 4521-33. 